\title{
Electroactive Properties and Electrochemical Stability of Poly(3,4-ethylenedioxythiophene) and Poly(N-methylpyrrole) Multi-layered Films Generated by Anodic Oxidation
}

\author{
Francesc Estrany ${ }^{a, *}$, Ramon Oliver ${ }^{a}$, Elaine Armelín ${ }^{b}$, José Ignacio \\ Iribaren ${ }^{b}$, Francisco Liesac, Carlos Alemán, \\ a) Unitat de Química Industrial, Escola Universitaria d'Enginyeria Técnica Industrial de \\ Barcelona, Universitat Politécnica de Catalunya, Comte d'Urgell 187, 08036 Barcelona, Spain \\ ${ }^{b)}$ Departament d'Enginyeria Química, E. T. S. d'Enginyers Industrials, Universitat Politécnica \\ de Catalunya, Diagonal 647, 08028 Barcelona, Spain \\ ${ }^{c}$ Departament d'Enginyeria Mecànica, E. T. S. d'Enginyers Industrials, Universitat Politécnica \\ de Catalunya, Diagonal 647, 08028 Barcelona, Spain
}

\begin{abstract}
Multi-layered films of poly(3,4-ethylenedioxythiophene), and poly(N-methylpyrrole), have been prepared using a layer-by-layer electrodeposition technique. The electrochemical properties and the conductivity of the films formed by 3,5 and 7 layers have been compared with their homopolymers and with the copolymers prepared from 3,4-ethylenedioxythiophene and N-methylpyrrole with different concentration ratios. The electroactivity and stability of the multi-layered systems were higher than those of PEDOT homopolymer and copolymers. Furthermore, the electrochemical properties improved when the number of layers increased. In addition, the electrical conductivity of the multi-layered films is higher than those of poly(N-methylpyrrole) and copolymers, and comparable to the PEDOT homopolymers. These results indicate that multi-layered systems show better performance than their homopolymers and/or copolymers derivatives for some applications like anticorrosive coatings or sensor materials.
\end{abstract}

Keywords: poly(3,4-ethylenedioxythiophene); poly(N-methylpyrrole); multi-layered films; conducting polymers; layer-by-layer electrodeposition.

\section{Introduction}

Poly(3,4-ethylenedioxythiophene), usually denoted PEDOT, is one of the most successful polythiophene derivatives because of its interesting properties: high

\footnotetext{
* Corresponding author. E-mail address: francesc.estrany@upc.edu , carlos.aleman@upc.edu
} 
conductivity, unusual electroactivity and a relative environmental stability [1-7]. On the basis of these properties, PEDOT derivatives are used in a wide range of electronic, electrochemical and optical applications [1 ]. In recent years, the properties of PEDOT have been combined with those of other typical conducting polymers to produce new copolymers with intermediate properties between both materials [8-15]. In particular, copolymers prepared by direct electrochemical oxidation from mixtures of 3,4-ethylenedioxythiophene (EDOT) with pyrrole [8,9], N-methylpyrrole (NMPy) [10], bithiophene [11], 3-methylthiophene [12], indole [13], and others, have provided materials with, in some cases, promising technological applications.

Very recently, we prepared the copolymer of EDOT and poly(N-methylpyrrole), or-PNMPy, hereafter denoted poly(EDOT-co-NMPy), via electrochemical oxidation on steel electrodes using monomer mixtures with various concentration ratios [10]. Comparison between poly(EDOT-co-NMPy) and the corresponding homopolymers revealed that, unfortunately, copolymers do not present any practical advantage with respect to PEDOT and (PNMPy) [10,16]. More specifically, the electroactivity of poly(EDOT-co-NMPy) is lower than that of both PEDOT and PNMPy homopolymers, i.e. the formation of electroactive polarons in the copolymers films is more difficult than those of homopolymers. Furthermore, the electrochemical stability of the homopolymers is significantly higher than that of the copolymer. Thus, redox properties of PEDOT and PNMPy are almost unaltered after eight consecutive oxidation-reduction cycles, while the degradation of the poly(EDOT-co-NMPy) properties is observed after only two consecutive oxidation-reduction cycles [10].

In this work, we present how all these deficiencies are successfully overcome by fabricating multi-layered films of PEDOT and PNMPy (ml-PEDOT/PNMPy) using a layer-by-layer electrodeposition technique. Systems containing 3, 5 and 7 layers, that alternate PEDOT and PNMPy, have been prepared and their electrochemical and electrical properties determined. In order to provide environmental stability to the multi-layered systems, PEDOT has been used for the outside layers. Results indicate that the electroactivity and electrochemical stability of ml-PEDOT/PNMPy are significantly higher than homopolymers generated under similar experimental conditions. Furthermore, the electrical conductivities of the ml-PEDOT/PNMPy prepared in this work were found to be closer to the PEDOT polymer. All the experimental results indicate that these multi-layered systems present a significant improved performance with respect to poly(EDOT-co-NMPy) copolymers, being considered very promising materials for some technological applications.

\section{Methods}

EDOT, NMPY and acetonitrile of analytical reagent grade were purchased from Aldrich and used as received without further purification. Anhydrous lithium perchlorate, analytical reagent grade, from Aldrich was stored in an oven at $80{ }^{\circ} \mathrm{C}$ before use in the electrochemical trials.

Homopolymers, copolymers and multi-layered films were prepared by chronoamperometry (CA) using a PAR 273A potenciostat-galvanostat connected 
to a PC computer and controlled through the PAR M270 program. All electrochemical experiments were conducted in a three-electrode twocompartment cell under nitrogen atmosphere $\left(99.995 \%\right.$ in purity) at $25{ }^{\circ} \mathrm{C}$. The working compartment was filled with $40 \mathrm{~mL}$ of a $10 \mathrm{mM}$ monomer solution in acetonitrile with $0.1 \mathrm{M} \mathrm{LiClO}_{4}$. The cathodic compartment was filled with $10 \mathrm{~mL}$ of the same electrolyte solution. Steel AISI 316 sheets of $4 \mathrm{~cm}^{2}$ area were employed as working electrodes, while a $2 \mathrm{~cm}^{2}$ area steel sheet was used as counter electrode. Before each trial, electrodes were cleaned with acetone and dried in an air-flow. The reference electrode was a $\mathrm{Ag} \mid \mathrm{AgCl}$ electrode containing a $\mathrm{KCl}$ saturated aqueous solution $\left(E^{\mathbf{o}}=0.222 \mathrm{~V} v s\right.$. standard hydrogen electrode at $25^{\circ} \mathrm{C}$ ), which was connected to the working compartment through a salt bridge containing the electrolyte solution. All potentials $(E)$ given in this work are referred to this electrode. The procedure used to prepare poly(EDOT-co-NMPy) was identical to that reported in our recent work [10].

The electrical conductivities of oxidized poly(EDOT-co-NMPy) and mlPEDOT/PNMPy systems were measured using the following method: a film of the material is taken off on a sticky double slide tape, that sticks as well on a little glass plate. Their ends are painted with conductive painting of colloidal silver, and later the measurement of their electrical resistance is made.

\section{Results and discussion}

\section{Preparation of multi-layered films}

Recently, the oxidation-polymerization processes of EDOT [16], NMPy [10] and EDOT:NMPy mixtures [10] were studied by cyclic voltammetry (CV) in the potential range from $-0.50 \mathrm{~V}$ to $1.80 \mathrm{~V}$ using a $10 \mathrm{mM}$ monomer solution of acetonitrile with $0.1 \mathrm{M} \mathrm{LiClO}_{4}$ and a $4 \mathrm{~cm}^{2}$ steel AISI 316 electrode. Our previous results indicated that the optimum potential to generate PEDOT, PNMPy and poly(EDOT-co-NMPy) is $1.40 \mathrm{~V}$. Accordingly, ml-PEDOT/PNMPy films were generated by CA under a constant potential of $1.40 \mathrm{~V}$.

Multi-layered films were generated using a layer-by-layer electrodeposition procedure. Initially, a layer of PEDOT was generated by immersion of the working electrode for a polymerization time of $100 \mathrm{~s}\left(\mathrm{t}_{\mathrm{pol}}\right)$ in a $10 \mathrm{mM}$ EDOT solution of acetonitrile with $0.1 \mathrm{M} \mathrm{LiClO}_{4}$. Next, the electrode coated with the PEDOT monolayer was immersed in a new cell filled with a $10 \mathrm{mM}$ NMPy solution of acetonitrile with $0.1 \mathrm{M} \mathrm{LiClO}_{4}$. A layer of PNMPy, the PEDOT monolayer by applying a constant potential of $1.40 \mathrm{~V} \mathrm{t}_{\mathrm{pol}}=100 \mathrm{~s}$. Next, a third layer of PEDOT was deposited on the electrode with the electrochemical condition described above for the first layer. Therefore, a 3-layered PEDOT/PNMPy/PEDOT film was obtained according to this method. Multilayered films containing 5 and 7 layers were prepared by using the same procedure. It should be mentioned that the adherence between PEDOT and PNMPy layers was excellent in all cases. The anodic current density stabilizes at about 1.7 and $2.0 \mathrm{~mA} \mathrm{~cm}^{-2}$ for PEDOT and PNMPy layers, respectively. Similar values were obtained for the ml-PEDOT/PNMPy films containing 5 and 7 layers. These high values, which are similar to those previously obtained for the generation of single-layered PEDOT and PNMPy films [10,16], indicate that the 
flow of monomers during the electrogeneration process is very high with respect to other thiophene derivatives previously studied [18-20].

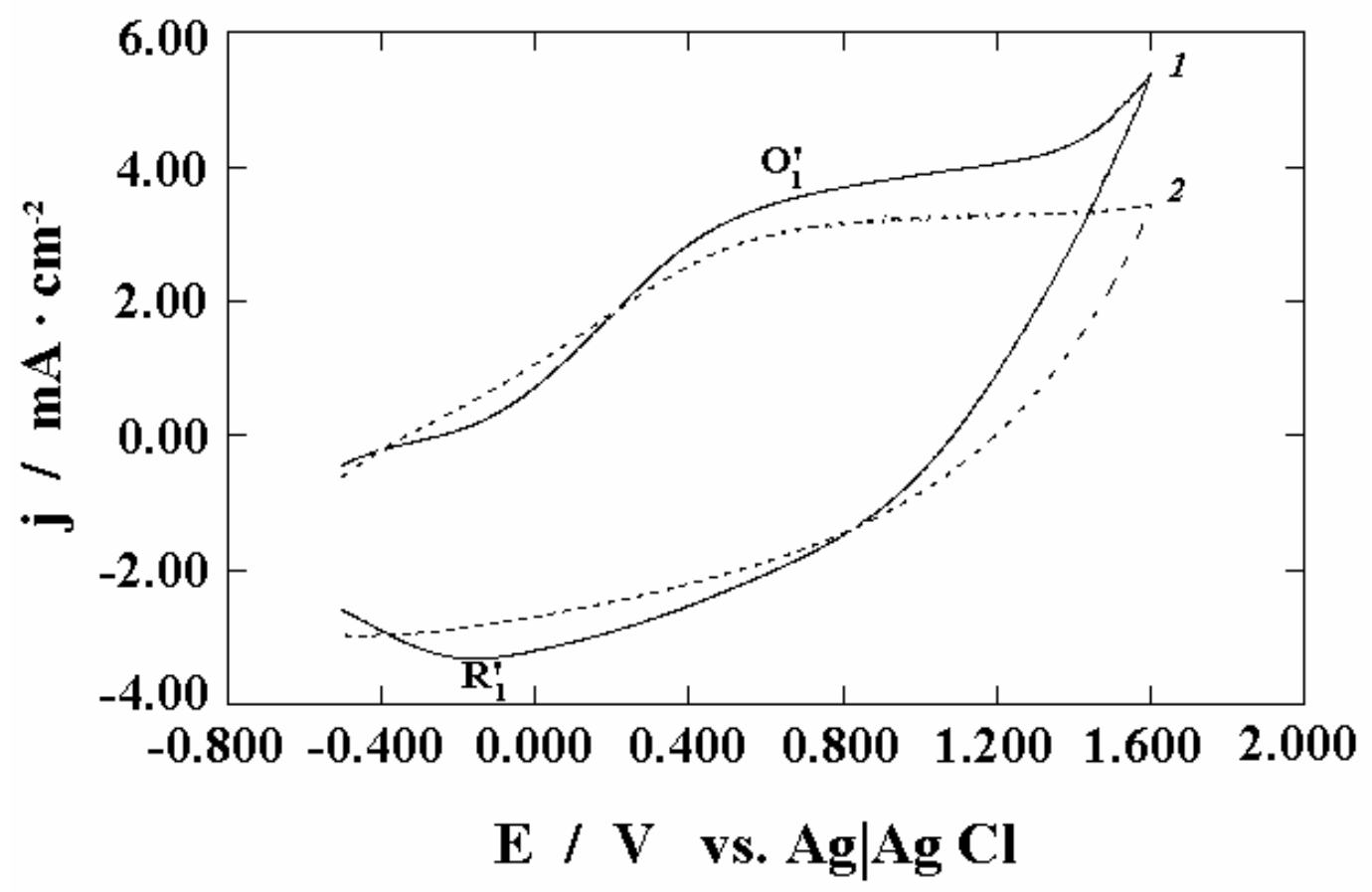

Figure 1. Control voltammograms for the oxidation of ml-PEDOT/PNMPy films with 3 layers (1) and PEDOT generated using a $t_{\mathrm{pol}}=300 \mathrm{~s}(2)$. The experimental conditions used to prepare both ml-PEDOT/PNMPy and pure PEDOT are described in the methods section. Voltammograms were recorded using a $4 \mathrm{~cm}^{2}$ steel electrode in acetonitrile with $0.1 \mathrm{M} \mathrm{LiClO}_{4}$, at $100 \mathrm{mV} \cdot \mathrm{s}^{-1}$ and $25{ }^{\circ} \mathrm{C}$. Initial and final potentials: $-0.500 \mathrm{~V}$; reversal potential: $1.600 \mathrm{~V}$.

\section{Electrochemical behaviour and control of multi-layered films}

Fig. 1 compares typical control voltammograms of PEDOT homopolymer films and ml-PEDOT/PNMPy film with 3 layers prepared as the procedure described above. It is worth noting that single-layer PEDOT film was generated by CA at $1.40 \mathrm{~V}$ during $300 \mathrm{~s}$, which is the same polymerization time of the mlPEDOT/PNMPy film with three layers. One prominent characteristic is the sharper oxidation and reduction curves for the ml-PEDOT/PNMPy film as compared to PEDOT. An oxidation shoulder, $\mathrm{O}_{1}$,', was observed, with anodic oxidation peak at $0.625 \mathrm{~V}$, and the corresponding reducction peak, $\mathrm{R}_{1}$, with $E_{\mathrm{p}}{ }^{\mathrm{c}}$ $=-0.155 \mathrm{~V}$, for the ml-PEDOT/PNMPy (3 layers). However, the cyclic voltammogram of single-layer PEDOT film did not present a clear shoulder for the oxidation or reduction curves. Nevertheless, both voltammograms showed that the oxidation processes can follow at higher potentials and the processes are reversible, i.e. the cathodic reduction process was observed in both cases. The cyclic voltammogram depicted in the Fig. 1 showed that the anodic and cathodic areas of ml-PEDOT/PNMPy film (3 layers) was remarkably higher. 


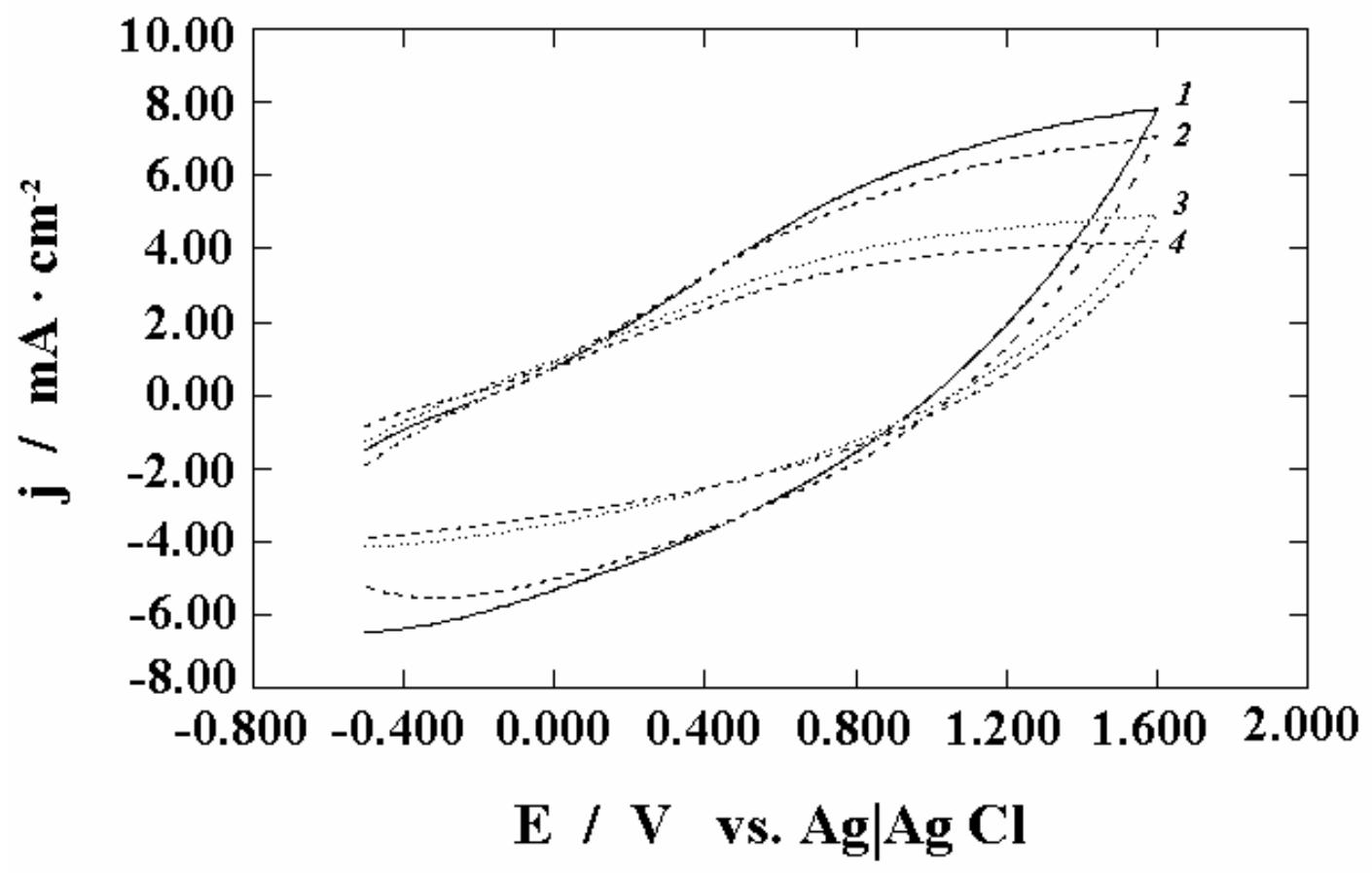

Figure 2. Control voltammograms for the oxidation of ml-PEDOT/PNMPy films with 5 layers (2) and - 7 layers (1), and PEDOT generated using a polymerization time of $500 \mathrm{~s} \mathrm{(4)}$ and $700 \mathrm{~s} \mathrm{(3).} \mathrm{Voltammograms} \mathrm{were} \mathrm{recorded} \mathrm{using} \mathrm{a} 4 \mathrm{~cm}^{2}$ steel electrode in acetonitrile with $0.1 \mathrm{M} \mathrm{LiClO}_{4}$, at $100 \mathrm{mV} \cdot \mathrm{s}^{-1}$ and $25^{\circ} \mathrm{C}$. Initial and final potentials: $0.500 \mathrm{~V}$; reversal potential: $1.600 \mathrm{~V}$.

Fig. 2 illustrates the cyclic voltammograms of PEDOT films and mlPEDOT/PNMPy films containing 5 and 7 layers. In this case, single-layer PEDOT films were generated at $\mathrm{t}_{\mathrm{pol}}$ of 500 and $700 \mathrm{~s}$ in order to compare with the 5- and 7- layered films, respectively. As can be seen in the Fig. 2, the cathodic and anodic areas of the ml-PEDOT/PNMPy and PEDOT increase considerably with the number of layers and the polymerization time, respectively, being this behavior specially appreciable for the multi-layered systems. Accordingly, it can be stated that the ability to store charge of mlPEDOT/PNMPy increases significantly with the number of layers. Although this ability is also enhanced when the polymerization time of the single-layer PEDOT films grows from 300 to $700 \mathrm{~s}$, the improvement is considerably larger when the number of layers of ml-PEDOT/PNMPy systems increases from 3 to 7 . These results reflect a favorable coupling effect between the electroactivities of PEDOT and PNMPy layers. Furthermore, it should be noted that the voltammograms of ml-PEDOT/PNMPy and PEDOT become less defined when the number of layers and the polymerization time, respectively, increase. 


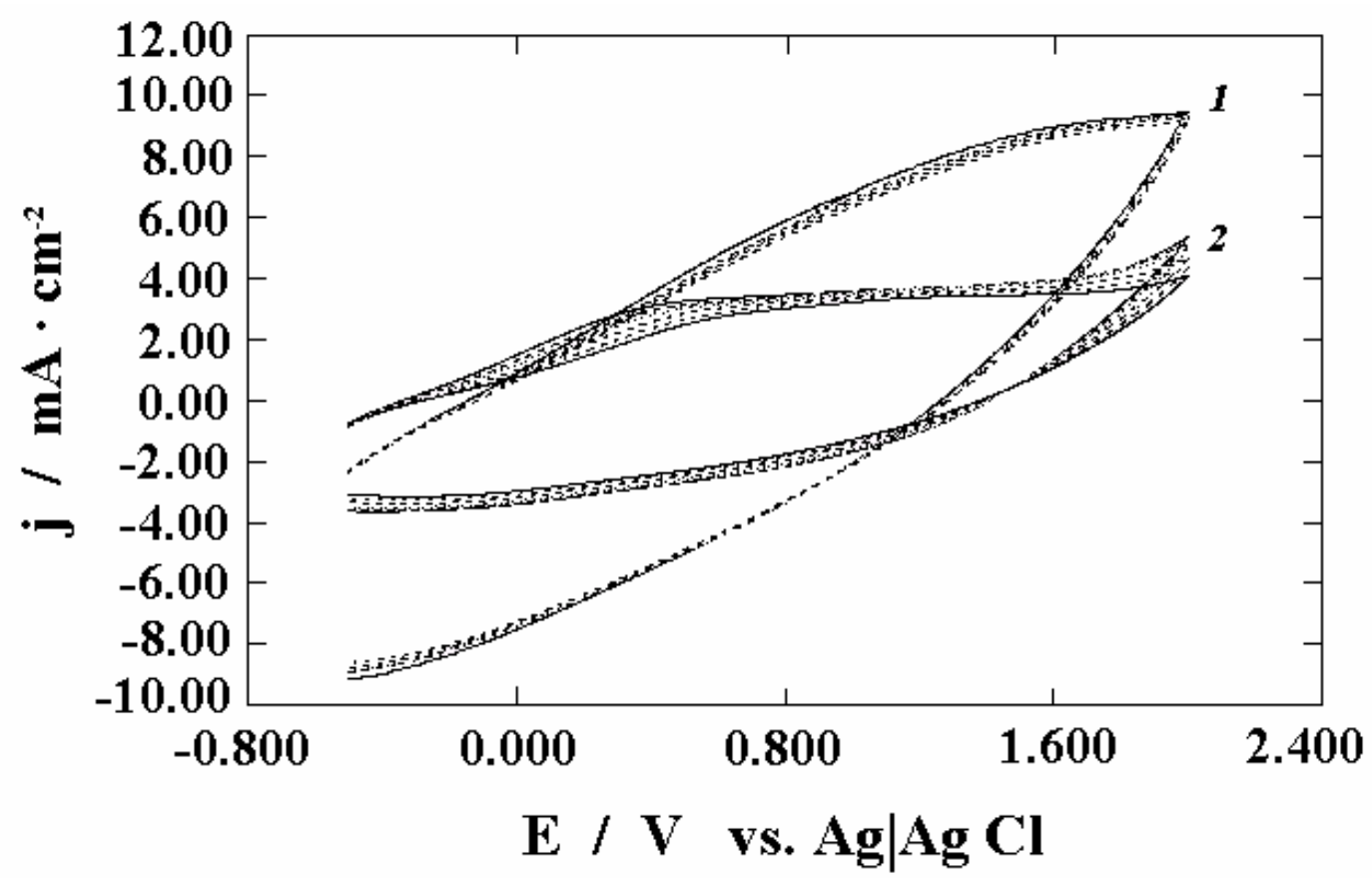

Figure 3. Control voltammograms for six consecutive oxidation-reduction cycles of mlPEDOT/PNMPy films with 7 layers (1), and PEDOT generated using a polymerization time of $300 \mathrm{~s}(2)$. Voltammograms were recorded using a $4 \mathrm{~cm}^{2}$ steel electrode in acetonitrile with $0.1 \mathrm{M} \mathrm{LiClO}_{4}$, at $100 \mathrm{mV} \cdot \mathrm{s}^{-1}$ and $25^{\circ} \mathrm{C}$. Initial and final potentials: $0.500 \mathrm{~V}$; reversal potential: $2.000 \mathrm{~V}$.

The electrochemical stability of the ml-PEDOT/PNMPy systems has been investigated by applying consecutive oxidation-reduction cycles. As above, results have been compared with those obtained for PEDOT generated using a $t_{\text {pol }}$ of $100 n \mathrm{~s}$, where $n$ is the number of layers of the multi-layered system used for the comparison. In all cases the electrochemical stability of the mlPEDOT/PNMPy was higher than that of pure PEDOT, the difference between such two systems growing with the number of layers of the multi-layered system. The electrochemical stability of single-layer PEDOT films is very remarkable at potentials lower than $1.80 \mathrm{~V}$. However, when they are oxidized at higher potentials, the oxidation and reduction areas of successive control voltammograms decrease gradually. This behaviour is reflected in the series 2 (Fig. 3), which shows six consecutive oxidation-reduction cycles recorded in the potential range from -0.50 to $2.00 \mathrm{~V}$ for single-layer PEDOT films generated applying $1.40 \mathrm{~V}$ during $300 \mathrm{~s}$. Furthermore, comparison of the results obtained using different polymerization times reveals that the reduction in the electroactivity of pure PEDOT is less apparent when the polymerization time is higher than $300 \mathrm{~s}$. Indeed, the electrochemical stabilities of the samples generated using 500 and $700 \mathrm{~s}$ are almost identical, as was found by determining the catodic and anodic areas of the corresponding voltammograms (available upon request to the authors). Accordingly, PEDOT homopolymer behaves asymptotically after polymerization times of $500 \mathrm{~s}$, even although the mass of polymer deposited in the electrode, i.e. the thickness of the film, increases with the polymerization time. The behavior of consecutive voltammograms of a 
PEDOT/PNMPy/PEDOT 3-layered film is similar to those of the PEDOT, since the oxidation and reduction areas also decrease with the number of cycles. However, in the case of PEDOT/PNMPy/PEDOT 5- and still more in case of 7layered film, the reduction of the areas of consecutive voltammograms is smaller. The series 1 (Fig. 3), that shows the voltammogram recorded for the 7-layered film, reveals how the electrochemical stability grows with the number of layers. It is worth noting that, in these cases, the voltammograms are almost overlapped. These results evidence that, in terms of electrochemical stability and electroactivity, the behavior of ml-PEDOT/PNMPy differs significantly from those of PEDOT and poly(EDOT-co-NMPy). Thus, the asymptotic behavior was not detected for the multi-layered system. On the other hand, the electrochemical stability of the copolymers is notably low, the integrated cathodic and anodic areas decreased after only two consecutive oxidation-reduction cycles [10]. Furthermore, the electroactivity of poly(EDOT-co-NMPy) was found to decrease when the concentration of NMPy increases. We can attribute this result to the fact that the electroactivity of PNMPy is considerably smaller than PEDOT [10], and it is possible to be interpreted that in copolymers there is an interaction between the monomer EDOT and the NMPy that affects negatively in a molecular scale the global electroactivity and the electrochemical stability of the copolymers. In the multi-layer systems, this negative interaction does not occur because the linkages are established between monomers with the same chemical composition, and the interactions between the homopolymers are promoted in a macroscopic scale.

\section{Conductivity of multi-layered films}

The influence of the number of layers in the electric properties of mlPEDOT/PNMPy system has also been subject of our study. Unfortunately, the films generated using $t_{\text {pol }}=100 \mathrm{~s} /$ layer were brittle and, in all cases, it was not possible to peel off the films from the electrode. In order to determine the conductivity of ml-PEDOT/PNMPy, new films with better mechanical properties of the 3-, 5- and 7-layered systems were generated layer-by-layer using the procedure described above but with a larger polymerization time. This way, the thickness of the layers was good enough to separate the films without breaking them. In all cases, the polymerization times used to generate the multi-layered systems were: a) $700 \mathrm{~s}$ for the first and the last PEDOT layers, i.e. the two external sides of the film; b) $500 \mathrm{~s}$ for the internal PEDOT layers; and c) $200 \mathrm{~s}$ for the PNMPy layers. The electrical conductivities $(\kappa)$ measured are listed in Table 1.

It is worth noting that the $\kappa$ of ml-PEDOT/PNMPy decreases slowly with the number of layers. Indeed, the reduction showed in Table 1 is almost negligible since in all cases the $\kappa$ values were within the same order of magnitude. On the other hand, the $\kappa$ determined for the films of PEDOT, films generated using polymerization times ranging from 1200 to $1500 \mathrm{~s}$, was an average of $160 \mathrm{~S} \mathrm{~cm}^{-1}$. Furthermore, the $\kappa$ values determined for PEDOT films are within the same order of magnitude as those measured for films generated using lower polymerization times, which was $210 \mathrm{~S} \mathrm{~cm}^{-1}$ for the films generated applying a constant potential 
of $1.40 \mathrm{~V}$ during $300 \mathrm{~s}$ [16]. Accordingly, as it was expected, the influence of the thickness of the films in the electric properties of PEDOT homopolymer is very small.

Table 1. Electrical conductivity ( $\kappa)$ of ml-PEDOT/PNMPy, PEDOT and PNMPy homopolymers and poly(EDOT-co-NMPy) copolymers studied in this work prepared with various EDOT:NMPy concentration ratios. ${ }^{\text {a }}$

\begin{tabular}{|c|c|}
\hline System & $\kappa\left(\mathrm{S} \mathrm{cm}^{-1}\right)$ \\
\hline ml-PEDOT/PNMPy (3 layers) & 79 \\
\hline ml-PEDOT/PNMPy (5 layers) & 45 \\
\hline ml-PEDOT/PNMPy (7 layers) & 24 \\
\hline PEDOT homopolymer ${ }^{\mathrm{a}}$ & $160-210$ \\
\hline PNMPy ${ }^{\mathrm{b}}$ homopolymer & $5.510^{-3}$ \\
\hline Poly(EDOT-co-NMPy) (EDOT:NMPY molar ratio 50:50) & $6.910^{-3}$ \\
\hline Poly(EDOT-co-NMPy) (EDOT:NMPY molar ratio 60:40) & $1.410^{-2}$ \\
\hline Poly(EDOT-co-NMPy) (EDOT:NMPY molar ratio 70:30) ${ }^{\mathrm{b}}$ & $7.310^{-1}$ \\
\hline $\begin{array}{l}\text { a Homopolymers and copolymers were prepared using the same ex } \\
\text { PEDOT/PNMPy: electrogeneration under a constant potential of } 1.40 \mathrm{~V} \mathrm{o} \\
\text { pure monomer (homopolymers) or mixture of monomers (copolymers) } \mathrm{s} \\
10 \mathrm{mM} \text { in acetonitrile with } 0.1 \mathrm{M} \mathrm{LiClO}_{4} \cdot \mathrm{t}_{\mathrm{pol}} \text { varied from } 300 \mathrm{~s} \text { to } 1500 \mathrm{~s} \text {. } \\
\mathrm{b}_{\mathrm{pol}}=300 \mathrm{~s} \text {. }\end{array}$ & $\begin{array}{l}\text { condition } \\
\text { teel electro } \\
\text { th a concen }\end{array}$ \\
\hline
\end{tabular}

The $\kappa$ values of PNMPy and poly(EDOT-co-NMPy) prepared with various EDOT:NMPy concentration ratios, have been also determined for comparison. For this purpose, films of the homopolymer and copolymers films were prepared using the experimental conditions previously described [10], by CA at $1.40 \mathrm{~V}$ during $300 \mathrm{~s}$ using a $10 \mathrm{mM}$ pure monomer (PNMPy) or mixture of monomers (copolymers) solution in acetonitrile with $0.1 \mathrm{M} \mathrm{LiClO}_{4}$. Three different EDOT:NMPy molar ratios, 50:50, 60:40 and 70:30, were considered in the polymerization medium, and the EDOT:NMPy molar ratios in the organic phase of the resulting copolymers being 25:75, 40:60 and 60:40, respectively. The $\kappa$ electrical conductivities measured for these films [10] are included in Table 1. It is worth noting that the $\kappa$ values of both PNMPy and the 25:75 copolymer are six and five orders of magnitude smaller than those of PEDOT and mlPEDOT/PNMPy systems, respectively. Furthermore, although the $\kappa$ increases significantly with the composition of EDOT present in the copolymer, it is still 
several orders of magnitude smaller than that determined for multi-layered systems.

The conductivity of the films composed by $3,-5$ and -7 layers was $79 \mathrm{~S} \mathrm{~cm}^{-1}$, $44.6 \mathrm{~S} \mathrm{~cm}^{-1}$ and $24.2 \mathrm{~S} \mathrm{~cm}^{-1}$, respectively. A study of the relationship between the conductivity and time was done. The behaviour of all the materials was very similar to the PEDOT homopolymer films, since they present a notable reduction between 50 and $55 \%$ the first 5 days, and later they tend to stabilize at $25 \%$ of initial value towards the 40-42 days of the beginning of the study. These results indicated that the content of PNMPy in the multi-layer polymers had a reducing effect in the conductivity of the material, but not so strong as the reducing effect detected for the copolymers in our previous work [10]. [The fact that the mlPEDOT/PNMPy conductivities show little more losses than the pure PEDOT is logical, since the current finds much greater resistance to its passage through the layers of PNMPy, being distributed through the PEDOT layers. As in a film to multi-layer the total straight section of PEDOT is always something inferior to that in a film of pure PEDOT with the same total thickness, the resistivity of the multi-layer film is always greater and, therefore, its conductivity is slightly smaller. Indeed, since this stability behavior time-conductivity has been determined in all the materials, we can conclude that the outside PEDOT layer gives a protective effect for the multi-layer systems, and therefore this PEDOT layer defines the temporary stability of the multilayer films.

\section{Conclusions}

Films of ml-PEDOT/PNMPy containing 3, 5 and 7 layers have been prepared using a layer-by-layer electrodeposition technique. In order to take advantage of the environmental stability of PEDOT, this polymer was used for the first and last layers of the generated systems. The electroactivity, electrochemical stability and electrical conductivity of the ml-PEDOT/PNMPy systems have been compared with those of homopolymers and poly(EDOT-co-NMPy) copolymers.

Electrochemical studies indicate that the ability to store charge of multi-layered films is higher than that of single-layer PEDOT films, in spite of the PEDOT ability to store charge is higher than that of PNMPy. Furthermore, this ability increases significantly with the number of layers and with the film thickness in the ml-PEDOT/PNMPy. Accordingly, the improvement of the latter systems with respect to pure PEDOT increases with the thickness of the films. On the other hand, the electrochemical stability of ml-PEDOT/PNMPy is considerably higher than that of poly(EDOT-co-NMPy), evidencing that films composed by alternate layers of PEDOT and PNMPy present significant advantages with respect to copolymers of EDOT and NMPy. Indeed, the electrochemical stability of multilayered systems, which increases with the number of layers, is higher than that of pure PEDOT, which does not depend on the thickness of the films after a threshold value.

Finally, the conductivities of ml-PEDOT/PNMPY, poly(EDOT-co-NMPy) and the corresponding homopolymers reveal that the values obtained for the multilayered systems are only one order of magnitude smaller than of PEDOT, which 
is the most conductive system investigated in this work. However, the copolymers present low conductivities, which are closer to that of PNMPy.

With regard to the stability of the conductivity with the time, the behaviour of all the multi-layers is very similar to pure PEDOT films: they have a reduction between 50 and $55 \%$ in the first 5 days, and later they have a tendency to estabilized at $25 \%$ of the initial value towards the 40-42 days of the beginning of the study. Therefore, in all the cases, the outer PEDOT layers of multi-layer films define its temporary stability.

The results reported in this work evidenced that ml-PEDOT/PNMPy films present significant advantages, especially in terms of electrochemical properties, not only with respect to poly(EDOT-co-NMPy), but also with respect to PEDOT. Thus, ml-PEDOT/PNMPy is expected to have better performance as protector against corrosion than pure PEDOT, even although the latter polymer was found to be an excellent anticorrosive additive for paints [20].

\section{Acknowledgements}

This work was supported by MCYT and FEDER funds with Grant MAT200300251.

\section{References}

1. B.L. Groenendaal, F. Jonas, D. Freitag, H. Pielartzik, J.R. Reynolds, $A d v$. Mater. 12 (2000) 481-494.

2. Q. Pei, G. Zuccarello, M. Ahlskog, O. Inganäs, Polymer 35, Iss. 7 (1994) 1347-1351.

3. X. Crispin, F.L.E. Jakobsson, A. Crispin, P.C.M. Grim, P. Andersson, A. Volodin, C. van Haesendonck, M. Van der Auweraer, W.R. Salaneck, M. Berggren, Chem. Mat. 18 (2006) 4354-4360.

4. D.W. Breiby, L.B. Samuelsen, B. Groenendaal, B. Struth, J. Polym. Sci.: Part B: Polym. Phys. 41 (2003) 945-952.

5. S.K.M. Jönsson, J. Birgersson, X. Crispin, G. Grezynsky, A.W.D. Osikowicz, W.D. van der Gon, W.R. Salaneck, M. Fahlman, Synth. Met. 139 (2003) 1-10.

6. B. Winther-Jensen, K. West, React. Funct. Polym. 66 (2006) 479-483.

7. S. Ghosh, O. Inganas, J. Electrochem. Soc. 147 (2000) 1872-1877.

8. G. Sönmez, A.S. Saraç, Synth. Met. 135/136 (2003) 459-460.

9. A.S. Saraç, G. Sönmez, F.Ç. Cebeci, J. Appl. Electrochem. 33 (2003) 295-301.

10. R. Oliver, A. Muñoz, C. Ocampo, C. Alemán, E. Armelin, F. Estrany, Chem. Phys. 328 (2006) 299-306.

11. N.M. Alpatova, E.V. Ovsyannikova, F. Jonas, S. Kirmeyer, E.Y. Pisarevskaya, M.Y. Grosheva, Russian J. Electrochem. 38 (2002) 576582.

12. T. Yohannes, J.C. Carlberg, O. Ingänas, T. Solomon, Synth. Met. 88 (1997) 15-21. 
13. J. Xu, G. Nie, S. Zhang, X. Han, J. Hou, S. Pu, J. Mat. Sci. 40 (2005) 2867- 2873.

14. Z.H. Wei, J.K. Xu, J. Hou, W.Q. Zhou, S.Z. Pu, J. Mat. Sci. 41 (2006) $3923-3930$.

15. V. Seshadri, L. Wu, G.A. Sotzing, Lagmuir 19 (2003) 9479 - 9485.

16. C. Ocampo, R. Oliver, E. Armelin, C. Alemán, F. Estrany, J. Polym. Res. 13 (2006) 193-200.

17. E. Brillas, J. Carrasco, R. Oliver, F. Estrany, J. Vilar, J.M. Morlans, Electrochim. Acta 45 (2000) 4049-4057.

18. F. Estrany, R. Oliver, E. García, E. Gualba, P.L. Cabot, E. Brillas, Collect. Czechl. Chem. Commun. 68 (2003) 1326-1344.

19. E. Brillas, R. Oliver, F. Estrany, E. Rodríguez, S. Tejero, Electrochim. Acta 47 (2002) 1623-1631.

20. F. Liesa, C. Ocampo, C. Alemán, E. Armelin, R. Oliver, F. Estrany, J. Appl. Polym. Sci. 102 (2006) 1592-1599. 